\section{$\underset{\substack{\text { hommes } \\ \text { \& migrations }}}{ }$}

\section{Hommes \& migrations}

Revue française de référence sur les dynamiques

migratoires

$1282 \mid 2009$

Santé et droits des étrangers : réalités et enjeux

\title{
Droit à la santé des ressortissants communautaires vivant en France
}

Les difficultés d'accès à la protection maladie et aux soins

\section{Antoine Math}

\section{(2) OpenEdition Journals}

Édition électronique

URL : http://journals.openedition.org/hommesmigrations/461

DOI : 10.4000/hommesmigrations.461

ISSN : 2262-3353

Éditeur

Musée national de l'histoire de l'immigration

Édition imprimée

Date de publication : 1 novembre 2009

Pagination : 122-135

ISSN : $1142-852 X$

Référence électronique

Antoine Math, «Droit à la santé des ressortissants communautaires vivant en France 》, Hommes \& migrations [En ligne], 1282 | 2009, mis en ligne le 29 mai 2013, consulté le 14 novembre 2019. URL http://journals.openedition.org/hommesmigrations/461; DOI : 10.4000/hommesmigrations.461 


\section{Droit à la santé des ressortissants communautaires vivant en France Les difficultés d'accès à la protection maladie et aux soins}

Par Antoine Math, chercheur en sciences sociales, Institut de recherches économiques et sociales (IRES)

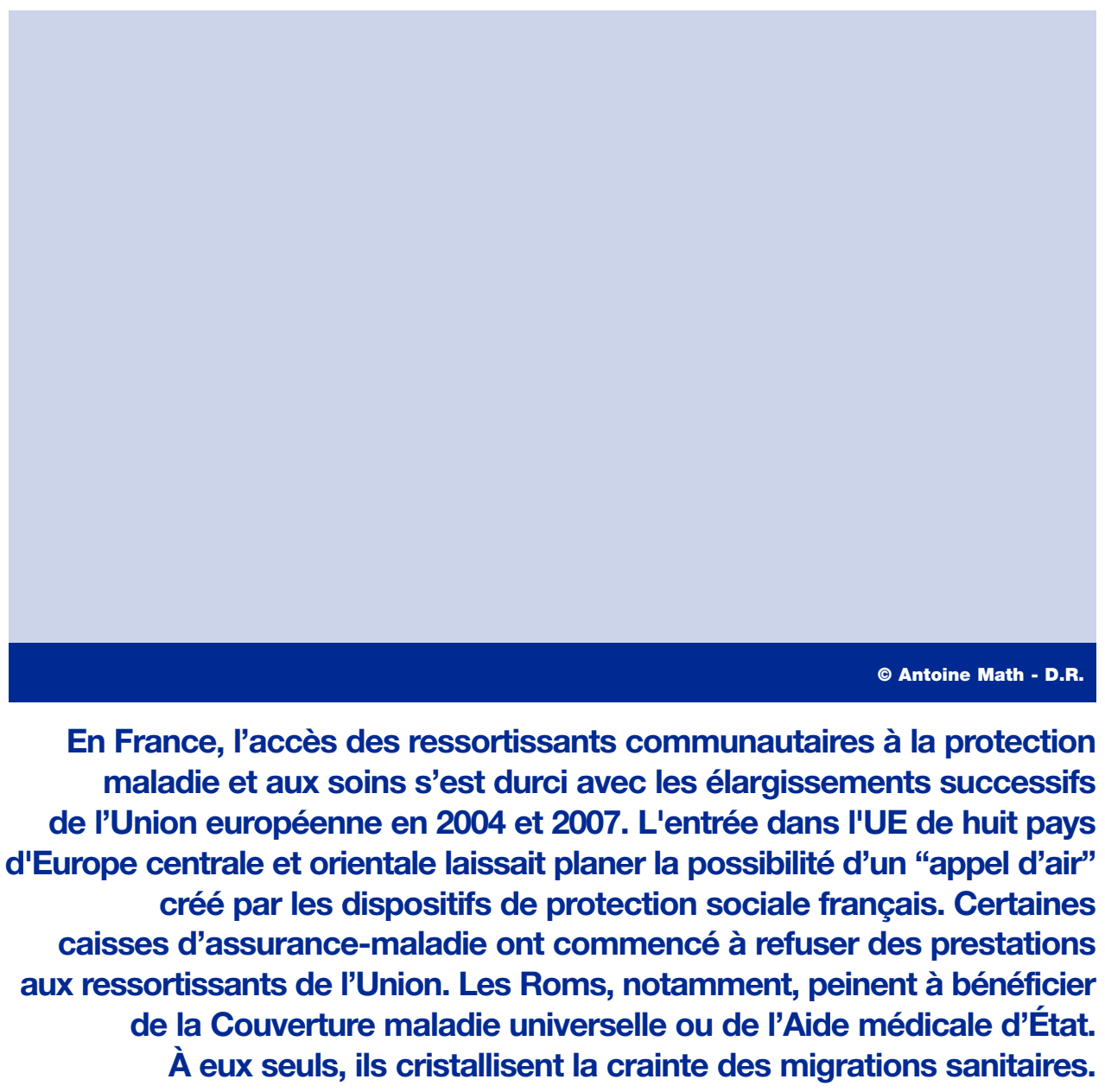


À l'occasion de la réforme de la CMU, se pencher sur le droit à une protection maladie et à l'accès aux soins des citoyens européens en France aurait présenté un intérêt très limitét ${ }^{(1)}$ À cette époque, l'égalité entre Français et ressortissants d'un pays membre de l'Union européenne (UE) ou ressortissants communautaires (RC) était largement devenue une réalité, dans les textes comme dans les pratiques. Des instructions officielles données au début des années deux mille préconisaient l'attribution de la CMU ou du RMI à tout RC vivant en France, sans qu'il ait à produire un titre de séjour ou d'autres pièces particulières. Ces textes reflétaient une évolution des mentalités ayant conduit à assimiler les RC aux nationaux. En retour, ils renforçaient la réalité du principe d'égalité entre citoyens européens. De fait, très peu de refus de prestations étaient signalés, à tel point que les associations défendant les droits des étrangers consacraient désormais leurs efforts aux $\mathrm{RC}$ dont les droits sociaux étaient bien mis à mal. Cette situation était l'aboutissement d'une lente évolution. Près d'un demi-siècle après la mise en place du Marché commun et de la liberté de circulation des travailleurs, les citoyens européens semblaient avoir enfin obtenu la reconnaissance effective de l'égalité des droits. L'attitude des pouvoirs publics français vis-à-vis des ressortissants communautaires en matière d'accès à la protection sociale s'est toutefois modifiée vers 2004, en lien manifeste avec l'élargissement de l'UE à des pays d'Europe centrale et orientale et l'arrivée, plus fantasmée que réelle, de citoyens pauvres symbolisés par la figure des Roms. D’une manière générale, ces ressortissants partagent les mêmes difficultés que les autres étrangers, voire d'autres personnes en situation de précarité. Ces dernières, y compris celles qui disposent d'une assurancemaladie, subissent aujourd'hui en France une dégradation de l'accès aux soins du fait de nombreux obstacles, financiers ou non ${ }^{(2)}$. Pour ceux qui ne peuvent se payer une assurance maladie complémentaire (ou "mutuelle") et qui doivent s'acquitter du ticket modérateur s'ajoutent les pratiques croissantes de dépassements d'honoraires, les difficultés pour bénéficier du tiers payant et l'augmentation des forfaits et autres franchises. Les refus de soins par des professionnels de santé sont en outre fréquents à l'égard des personnes les plus démunies, a fortiori celles dont la situation de forte précarité est signalée par le type de couverture maladie (CMU C, AME).

\section{La longue et difficile marche européenne vers l'égalité des droits}

L'histoire des discriminations en matière de protection sociale est aussi longue que l'histoire de la protection sociale ${ }^{(3)}$. Sous la pression de la jurisprudence de la Cour de justice des communautés européennes (CJCE), dont le contentieux sur les questions 
de sécurité sociale est très volumineux, les discriminations à l'encontre des $\mathrm{RC}$ vont peu à peu être censurées. Le mouvement de prohibition des discriminations a d'abord bénéficié aux travailleurs communautaires pouvant se prévaloir de la liberté de circulation. Il s'agissait d'abord des discriminations directes dites "ostensibles", c'està-dire correspondant à une différence de traitement fondée sur une caractéristique précise comme la nationalité (ou le sexe). L'égalité de droit entre nationaux et RC en matière sociale n'était pas un objectif en soi de la Communauté économique européenne: elle va d'abord s'imposer comme un moyen de rendre effectif le marché unique des travailleurs inscrit dans le traité de Rome.

Il importait, en effet, de faire en sorte que les différences entre les régimes de sécurité sociale ne puissent pas constituer une entrave pour les travailleurs migrants voulant utiliser leur droit d'exercer une activité professionnelle sur le territoire d'un autre État. Des règlements communautaires ont été adoptés en ce sens au début des années soixantedix. Parmi les principes visant à ne pas pénaliser le travailleur exerçant son droit à la libre circulation figure l'égalité de traitement avec les ressortissants de l'État dans lequel le travailleur migrant exerce son activité. Ce mouvement vers l'égalité s'est étendu au-delà des seuls salariés, à toute personne obtenant un droit à la libre circulation (membres de famille, travailleurs indépendants, anciens travailleurs, étudiants, etc.). La CJCE, en se fondant sur la nouvelle citoyenneté européenne, a été jusqu'à juger que l'égalité de traitement en matière de protection sociale devait également s'appliquer, dans certaines situations, à des ressortissants inactifs ne retirant pas du traité un droit à la libre circulation. 
L'égalité des droits n'est donc plus seulement considérée comme un instrument pour parvenir à des objectifs économiques mais est devenue, en tant que telle, partie intégrante du projet européen, l'une de ses valeurs. La jurisprudence et les règlements communautaires n'ont cependant pas suffi pour parvenir immédiatement à l'égalité effective des droits. Réticents à adapter leurs législations et leurs pratiques, les États ont fait preuve de résistance. Plus récemment, l'objectif d'égalité a aussi intégré la lutte contre les discriminations indirectes, c'est-à-dire les dispositions ou pratiques apparemment neutres au regard du critère considéré - ici la nationalité -, mais qui ont pour effet d'affecter une proportion plus élevée de personnes d'une même catégorie. La CJCE a ainsi condamné de nombreuses discriminations indirectes à l'encontre des ressortissants communautaires dans le champ de la protection sociale : elle a ainsi admis que le lieu d'origine ou le domicile d'un travailleur, une durée de résidence préalable ou encore la résidence du conjoint sur le territoire de l'État pour obtenir une prestation à taux majoré pour les couples étaient des critères violant le principe d'égalité. De même a été prohibée l'exigence d'un titre de séjour qui, de fait, constituait un moyen pour les États de ne pas accorder des droits, ou de les attribuer avec retard. La notion de discrimination indirecte a été reprise dans les nouvelles directives européennes, adoptées à la suite du traité d'Amsterdam, puis progressivement intégrées dans les législations nationales. Ainsi, de nombreuses dispositions sociales ont dues être modifiées pour les RC.

\section{Au tournant des années deux mille, un citoyen européen enfin reconnu}

Cette évolution de la jurisprudence et du droit positif s'est accompagnée, tardivement mais sûrement, d'une évolution des mentalités qui a conduit peu à peu à une assimilation dans le traitement des nationaux et des RC. Au tournant des années deux mille, les autorités se montraient particulièrement bienveillantes. Il s'agissait aussi d'une attitude pragmatique. La libre circulation avait été étendue à de nombreuses catégories, y compris aux inactifs (étudiants, retraités...), le principe d'égalité était défendu et reconnu par les juges, et surtout l'exigence d'un titre de séjour par une caisse était désormais interdite.

S'échiner à déterminer parmi les nombreux RC vivant en France les rares qui ne disposaient pas d'un droit au séjour devenait une opération fastidieuse et coûteuse pour les caisses, mais aussi particulièrement inopportune dans un contexte de célébration de la toute nouvelle citoyenneté européenne. La mise en place de la carte européenne d'assurance-maladie participait également de ce climat favorable. 
Comme le dira crûment une circulaire ministérielle de 2007, à cette époque "la question de la régularité de leur séjour [et leur droit à l'assurance-maladie] était considérée comme a priori résolue ${ }^{(4)}$.

Après une longue période de défiance et de résistance pendant laquelle les administrations avaient cherché à limiter l'égalité effective des droits en matière de protection sociale, l'heure était donc à l'égalité, à l'assimilation. Au début des années deux mille, des instructions préconisaient l'attribution de la CMU ou du RMI, dans les mêmes conditions que pour les Français, à tout ressortissant communautaire vivant en France sans qu'il ait à produire de titre de séjour.

Ainsi, la circulaire ministérielle CMU du 3 mai 2000 précisait que "pour leur affiliation à un régime de sécurité sociale, notamment au régime général sur critère de résidence, [la] condition [de régularité de séjour] n'est en aucun cas opposable aux ressortissants de l'Union européenne et de l'Espace économique européen et à leurs ayants droit, quelle que soit la nationalité de ces derniers ${ }^{(5)}$. . Une instruction de la CNAM du 27 février 2006 précisait que même la condition de stabilité de la résidence en France (durée préalable de résidence d'au moins trois mois) pour l'accès à la CMU devait être

Cependant, en lien avec l'entrée dans l'UE de huit pays d'Europe centrale et orientale au $1^{\text {er }}$ mai 2004, de nouvelles pratiques ont abouti à des refus de prestations par certaines caisses de Sécurité sociale, là où, auparavant, les droits étaient accordés. assouplie pour les RC qui "ont la possibilité d'être affiliés à la CMU de base et/ou complémentaire sils cumulent trois mois de cotisations ou de résidence dans l'Union européenne ${ }^{(6) "}$.

Autre signe du climat bienveillant régnant à l'égard des RC, les associations de défense des droits des étrangers signalaient très peu de problèmes $^{(7)}$. Les Européens se voyaient d'ailleurs réserver une portion de plus en plus congrue dans les guides et autres brochures sur les droits sociaux, voire en disparaissaient totalement. L'incontournable guide du Comité médical des exilés, dans ses parties "accès aux soins" ou "protection maladie", ne donne ainsi aucune information spécifique concernant les RC. Sans le dire explicitement, ce guide est destiné aux étrangers non communautaires ${ }^{(8)}$. Il est aussi révélateur que les analyses informées produites sur l'état du droit à la protection maladie ou l'accès aux soins des étrangers en France n'abordent pas le cas des $\mathrm{RC}^{(9)}$.

$\mathrm{Si}$, ici ou là, certains citoyens européens pouvaient encore rencontrer des difficultés pour obtenir une couverture maladie, ils bénéficiaient, dans l'ensemble, d'une situation très favorable, notamment en comparaison des ressortissants d'États tiers. La question ne semblait plus faire problème. 


\section{L'élargissement de l'Union européenne : le déclenchement du backlash pour les citoyens européens ?}

Cependant, en lien avec l'entrée dans l'UE de huit pays d'Europe centrale et orientale au $1^{\text {er }}$ mai 2004, de nouvelles pratiques ont abouti à des refus de prestations par certaines caisses de sécurité sociale, là où, auparavant, les droits étaient accordés. L'élargissement de l'UE a été vite perçu comme un danger. C'est l'époque où émerge aussi le thème du "plombier polonais" venu travailler pour rien et prendre les emplois des locaux. Dans ce contexte, la crainte est que la protection sociale ne crée un "appel d'air". Il s'agit d'éviter les migrations sanitaires et de repousser les "pouilleux de l'Est", à commencer par les Roms, figures désormais emblématiques du parasite. La perspective de l'entrée dans l'UE au $1^{\text {er }}$ janvier 2007 de la Bulgarie et de la Roumanie, deux États plus pauvres encore que les précédents et disposant d'une importante minorité rom, a attisé ces phobies et précipité le changement d'attitude. Désormais, foin de l'égalité des droits, la protection sociale devait coûte que coûte être limitée et mise au service de la maîtrise des flux migratoires, comme elle l'est déjà largement s'agissant des étrangers non communautaires. Ce nouveau climat de défiance, qui n'est pas propre à la France, est dénoncé par le Parlement européen ${ }^{(10)}$. Premier signe tangible du changement de cap, une circulaire ministérielle du 24 mars 2005 est venue préciser - en pratique, limiter - l'accès des RC au RMI. Des restrictions supplémentaires ont été introduites dans la législation française en 2006 pour le RMI et en 2007 pour l'allocation de parent isolé, l'allocation aux adultes handicapés et 
la CMU. Portant sur des situations marginales, ces modifications législatives avaient surtout pour but de produire des effets d'annonce bien davantage que d'avoir un impact réel direct. Elles témoignent néanmoins du changement d'attitude des autorités françaises vis-à-vis des $\mathrm{RC}^{(11)}$.

À côté de ces changements législatifs, on a constaté que les caisses, de manière très variable d'un endroit à l'autre, modifiaient leurs pratiques, refusant de plus en plus souvent des prises en charge de maladies de façon arbitraire, abusive ou illégale, souvent de manière discriminatoire selon la nationalité du ressortissant communautaire. Des traitements différenciés ont été constatés, par exemple selon que le citoyen européen est Britannique, Roumain, a fortiori s'il est Rom. Ces derniers, surtout à partir de l'entrée de la Bulgarie et de la Roumanie dans l'UE, se trouvaient manifestement dans le collimateur des autorités ${ }^{(12)}$. Une instruction postée sur le site de la CNAM le 24 septembre 2007 illustre à merveille cette volonté de distinguer les RC selon leur nationalité : elle s'intitulait "Point d'information : affiliation à la CMU pour les ressortissants britanniques inactifs".

\section{Des règles normalement identiques pour tous les ressortissants communautaires}

Pourtant, en ce qui concerne l'examen des droits à la protection sociale et l'affiliation à l'assurance-maladie, aucune différence ne peut être faite entre un Britannique et un Bulgare. Les élargissements de l'Union européenne en 2004 et 2007 n'ont eu absolument aucune incidence sur le droit à la protection sociale applicable aux RC, ce que rappellent certaines circulaires ${ }^{(13)}$. La "période transitoire" appliquée aux ressortissants des nouveaux États membres vaut uniquement pour l'accès au travail salarié (depuis le $1^{\text {er }}$ juillet 2008, seuls les Bulgares et les Roumains sont encore concernés par cette restriction) et ne trouve aucune application en matière de protection sociale. Contrairement à certaines croyances, rien en droit ne justifiait un changement d'attitude. Rien n'est venu modifier les règles applicables aux ressortissants communautaires en matière de protection sociale : ni l'entrée de dix nouveaux États dans l'UE au $1^{\text {er }}$ mai 2004, ni l'entrée de la Roumanie et de la Bulgarie dans l'UE au $1^{\text {er }}$ janvier 2007, ni la directive 2004/38 relative au droit des citoyens de l'Union, ni la loi n²006-911 sur l'immigration du 24 juillet 2006, ni plus généralement les évolutions du droit communautaire. Pourtant, ces évènements ont fourni le prétexte à des changements de pratiques. Ainsi, une instruction de la CPAM du 93 de février 2007 se fondait sur l'entrée dans l'UE de la Roumanie et de la Bulgarie pour exclure de toute 
protection maladie les résidents inactifs de ces pays, jusque-là bénéficiaires de l'AME : non seulement les RC n'avaient plus droit à la CMU au motif de leur séjour irrégulier, mais on les excluait aussi de l'AME parce que, étant Européens, ils avaient le droit de venir en France et ne pouvaient donc se trouver en situation irrégulière... Kafka n'était pas loin.

Un "Point CMU" n 73 du 2 août 2007 diffusé par la CNAM s'appuyait sur la loi Sarkozy pour valider la nouvelle position consistant à exclure les inactifs du bénéfice de la CMU, même ceux qui en bénéficiaient depuis des années. Heureusement, cette instruction prévoyait de renvoyer les exclus de la CMU vers l'AME ou vers une prise en charge au titre du Fonds soins urgents (article L. 254-1 code de l'action sociale et des familles). Le "Point d'information" précité destiné aux Britanniques ainsi qu'un point CMU spécial de la CNAM du 28 septembre 2007 préconisaient de maintenir la CMU pendant un délai de grâce limité au maximum à six mois pour ceux qui en bénéficiaient déjà auparavant. Ce contexte d'absence d'instructions stables a favorisé des pratiques mouvantes et différenciées d'une caisse à l'autre.

\section{Le droit au maintien à une couverture maladie}

Les instructions de la CNAM ont été vivement contestées, en particulier par d'éminents spécialistes du droit communautaire comme le professeur Jean-Philippe Lhernould. Finalement, il a fallu attendre une circulaire ministérielle du 23 novembre 2007 pour avoir la première instruction de l'État en matière d'attribution de la $\mathrm{CMU}^{(14)}$. Cette circulaire revient sur la dernière instruction de la CNAM en ce qui concerne le maintien de l'affiliation à l'assurance-maladie pour les RC bénéficiant déjà de la CMU. En effet, même si cette affiliation a pu leur être accordée à tort, et même s'ils n'en rempliraient pas les conditions s'ils la demandaient aujourd'hui pour la première fois, "revenir sur cette affiliation reviendrait à remettre en cause un droit au séjour qui leur a été de facto reconnu”. Aussi, le ministère préconise aux CPAM "dans un premier temps, d'examiner les situations de ces personnes afin de déterminer si elles [ne peuvent pas être couvertes à un autre titre]" et "lorsque cet examen, au cas par cas, ne permet pas l'octroi d'une couverture médicale, les personnes concernées seront maintenues à l'assurance-maladie française via la CMU de base”.

Ce faisant, le ministère se plie aux exigences posées par la jurisprudence communautaire. Une administration ayant déjà reconnu à un RC le droit à la prestation lui a donc aussi reconnu de facto un droit de séjour, même si la personne 
ne répondait pas aux conditions pour en bénéficier : elle est liée par sa décision passée. Cette circulaire n'a pas tout réglé puisque certaines caisses ignorent la circulaire ministérielle et appliquent toujours les instructions précédentes de la CNAM en renvoyant les personnes vers l'AME. D'autres caisses s'ingénient aussi à considérer les demandes de renouvellement de la CMU, chaque année, comme de nouvelles demandes, notamment quand les demandes sont déposées après l'échéance, ce qui, là encore, conduit à exclure les personnes de la CMU et à les faire basculer, dans le meilleur des cas, vers l'AME.

\section{Les refus de CMU ou la mise à mal du principe d'égalité}

Le principe d'égalité garanti par les traités communautaires interdit les différences de traitement en matière de prestations sociales entre un RC et un Français. Si quelques restrictions sont autorisées, le seul obstacle réel demeure, en pratique, l'exigence d'un droit au séjour ${ }^{(15)}$. Pour vérifier cette condition, des caisses de protection sociale demandent encore très souvent la production d'un titre de séjour. Or les RC ne sont pas tenus - et ce depuis très longtemps en matière de protection sociale - de présenter un titre de séjour. Exiger pour l'octroi d'un droit social la présentation d'un titre de séjour ou, ce qui revient au même, tout autre "papier" délivré par la préfecture, est une exigence contraire au droit communautaire. Tout justificatif attestant d'un droit au séjour au regard des règles communautaires est suffisant pour accéder aux prestations. Et il revient aux caisses de protection sociale d'examiner les conditions d'attribution des prestations, y compris la régularité du séjour.

Alors même que l'exigence d'un titre de séjour pour l'affiliation à l'assurancemaladie est abusive, à l'inverse, un RC qui produit un titre de séjour a droit aux prestations à égalité avec les Français, et ce même s'il n'a jamais formellement rempli ou ne remplirait plus les conditions d'un droit au séjour. Reste tous les RC ne détenant pas de titre de séjour, situation de plus en plus fréquente puisque les RC ne sont plus tenus d'en détenir un au regard des règles du séjour, à l'exception des seuls Bulgares et des Roumains voulant exercer une activité professionnelle.

La règle de base est que tout RC ayant un droit au séjour a droit, à égalité avec les Français, à l'assurance-maladie. Il s'agit d'abord de tous les actifs, c'est-à-dire les personnes exerçant une activité professionnelle salariée ou non-salariée ou se trouvant dans une situation assimilée (chômeurs indemnisés, malades, etc.) permettant l'affiliation à l'assurance-maladie. Contrairement à des idées reçues et à l'interprétation 
faite par de nombreuses caisses de sécurité sociale, tous les inactifs n'ont pas à remplir la condition de ressources suffisantes et de couverture sociale préalable pour bénéficier d'un droit au séjour et donc des droits sociaux à égalité avec les nationaux. Outre ceux qui détiennent un titre de séjour, plusieurs catégories d'inactifs disposent d'un droit inconditionnel au séjour et ont droit à la CMU : ceux qui ont acquis un droit au séjour permanent après cinq ans de résidence régulière; ceux qui n'exercent plus d'activité salariée ou non-salariée mais qui conservent la qualité de travailleur salarié ou non-salarié; les membres de la famille d'un actif communautaire ou membres de la famille du titulaire d'un droit au séjour permanent.

Les autres inactifs qui bénéficient d'un droit au séjour, parce qu'ils en remplissent les conditions (ressources, couverture sociale) au moment de leur demande de CMU, ne peuvent se la voir refuser par principe, dès lors qu'ils justifient de la condition de résidence de trois mois préalable exigée pour l'accès à la CMU. Ainsi, l'inactif présent sur le territoire depuis plus de trois mois, justifiant de ressources suffisantes et d'une couverture sociale (régime français de sécurité sociale, régime étranger de sécurité sociale ou assurance privée), c'est-à-dire remplissant les conditions pour bénéficier d'un droit au séjour, peut, à partir

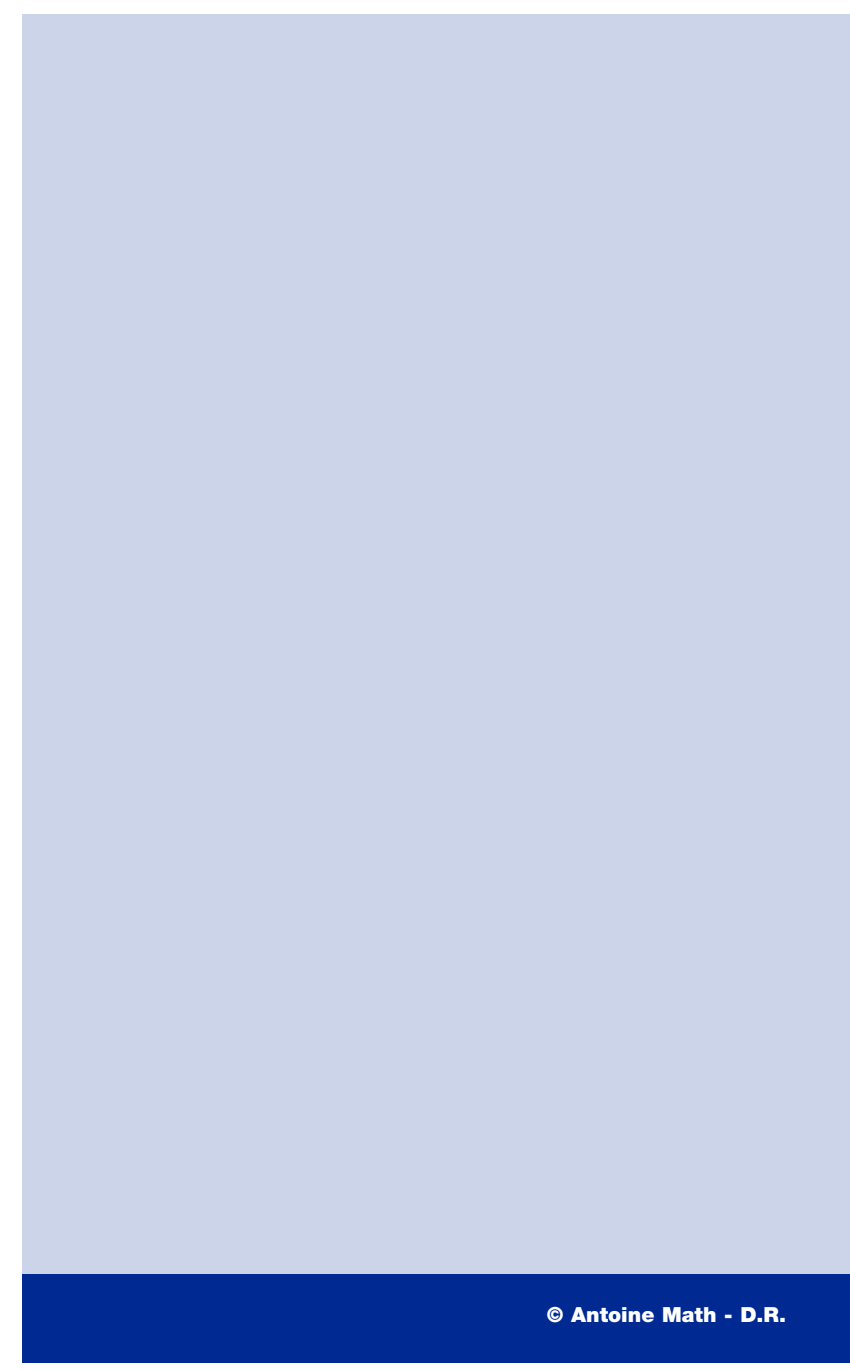
du quatrième mois de résidence, demander la CMU de base selon le critère de résidence stable et régulière, car il en remplit alors toutes les conditions ${ }^{(16)}$. Enfin, la CJCE, qui veille à ce que toute dérogation aux libertés fondamentales des citoyens européens, notamment au principe d'égalité, soit interprétée de manière 
très stricte, a jugé que même un inactif ne justifiant pas de ressources suffisantes ou d'une couverture sociale ne devait pas forcément être exclu des droits sociaux, en particulier s'il avait par le passé rempli les conditions d'un droit au séjour.

Alors que l'exclusion des RC du droit à la CMU devrait être assez exceptionnelle, la circulaire ministérielle du 23 novembre 2007 relative à l'accès des ressortissants communautaires à la CMU affirme une

Les restrictions à l'accès

à l'assurance-maladie, destinées initialement

à dissuader et pénaliser les Roms, tendent désormais à s'appliquer à tous les ressortissants communautaires et à s'étendre à l'accès à I'AME. inaccessibilité de principe à la CMU de base. Cette position est particulièrement contestable au regard du droit communautaire ${ }^{(17)}$ puisqu'elle aboutit à refuser la CMU de base aux RC inactifs - même ceux qui remplissent toutes les conditions pour en bénéficier (trois mois de séjour avec ressources et couverture sociale) - et à les renvoyer vers l'assurance privée. La circulaire revient aussi à traiter les ressortissants communautaires plus défavorablement que les non communautaires. Le renvoi vers des assureurs privés pour la couverture maladie de base pose d'autres problèmes : cette solution n'est pas possible pour tous puisque les assureurs privés opèrent des sélections selon le risque en excluant notamment les personnes malades ou âgées; cette solution, lucrative pour les assureurs privés, constitue, en outre, un manque à gagner préjudiciable aux finances publiques françaises puisque la CMU de base est versée moyennant le versement d'une cotisation ( $8 \%$ pour tous les revenus supérieur au plafond). Les restrictions à l'accès à l'assurance-maladie, destinées initialement à dissuader et pénaliser les Roms, tendent désormais à s'appliquer à tous les RC et à s'étendre à l'accès à l'AME.

\section{Des parias européens privés illégalement de l'AME}

Bien que des instructions de la CNAM et du ministère aient précisé que les RC ne remplissant pas la condition de séjour et exclus de l'assurance-maladie pouvaient prétendre à l'AME, les refus d'AME se sont poursuivis sous couvert de motivations diverses, souvent fantaisistes, surtout s'agissant de Roms. L'AME doit pourtant être accordée à toute personne disposant de faibles ressources et résidant en situation irrégulière en France depuis plus de trois mois (et même sans délai s'agissant des mineurs). Pouvoir justifier d'une résidence en France depuis plus de trois mois 
n'est déjà pas évident quand on vit dans des conditions d'habitat précaire qui ne permettent pas d'obtenir des justificatifs de résidence. Les intéressés doivent aussi composer avec les refus de certaines associations de fournir une attestation de la présence depuis plus de trois mois. Cette justification est d'autant plus difficile pour des Européens qu'ils sont entrés librement, sans formalités, sur le territoire français, sans laisser aucune trace sur leur passeport. Les caisses posent en outre des exigences abusives, telle la preuve de la résidence dans les jours précédents juste la date de trois mois antérieure à la demande, et refusent une demande avec des justificatifs de présence à trois mois et demi et deux mois... Les caisses posent également des exigences d'authenticité et de validité des pièces des plus farfelues. Ces tracasseries se répètent parfois lors du renouvellement annuel de l'AME, ce qui conduit à des pertes de droits.

Plus généralement, les demandeurs font aussi face à des démarches lourdes, plus longues que pour les sans-papiers non communautaires. On exige d'eux une procédure de domiciliation, même quand ils peuvent justifier d'une adresse postale pour recevoir leur courrier, et alors même qu'ils ne sont donc pas légalement sans résidence stable $^{(18)}$. Face aux refus des centres communaux d'action sociale de remplir leur obligation de domiciliation, nombreux sont ceux privés de droit pour ce seul motif. On signale aussi des services sociaux des maternités qui "oublient" d'informer du droit à l'AME dès le premier jour de présence pour les enfants. Des bébés nés en France se retrouvent ainsi sans couverture maladie. On constate aussi des refus de prise en charge d'enfants à charge d'un bénéficiaire de l'AME au motif illégal qu'aucun lien juridique n'existe entre l'adulte et le mineur.

\section{Les Roms, des citoyens européens plus mal traités que des sans-papiers non communautaires}

Dans certaines caisses, pour bénéficier de l'AME, il est également demandé aux RC de prouver qu'ils ne bénéficient pas d'une couverture médicale dans leur pays, ce qu'on ne leur demandait pas, s'agissant des Roumains et des Bulgares, avant que leur pays entre dans l'UE en 2007, ce qu'on ne demande toujours pas aux ressortissants non communautaires... Désormais exclus de la CMU, les RC se voient donc plus mal traités que des sans-papiers non européens en matière d'accès à l'AME. Ces exigences à l'égard des Roms roumains sont d'autant plus abusives qu'ils ne sont quasiment jamais couverts dans leur propre pays où leurs difficultés d'accès aux soins sont par ailleurs bien connues. Pire, des caisses refusent de façon contestable des attestations 
sur l'honneur de non-affiliation. De longues vérifications systématiques de la nonaffiliation des personnes en Roumanie doivent alors être engagées, ce qui prend parfois cinq ou six mois. Pour les Roms qui sont partis avec l'ANAEM dans le cadre d'une procédure de retour prétendument "volontaire" ${ }^{(19)}$, puis qui sont revenus, les caisses refusent également l'AME sur le seul fondement qu'ils ont reçu une aide de l'ANAEM. Cette exclusion de l'AME, non fondée en droit, est particulièrement critiquable, s'agissant de mineurs de surcroît.

Toutes ces mesures dissuasives ou dilatoires conduisent les Roms et leurs enfants, privés de protection maladie, à devoir renoncer aux soins ou bien à se rendre à l'hôpital, aux urgences ou dans les Permanences pour l'accès aux soins santé(PASS), engorgeant inutilement certains services hospitaliers.

En dernier recours, les exclus de toute protection maladie devraient pouvoir bénéficier d'une prise en charge des soins reçus à l'hôpital à travers le Fonds pour les soins urgents et vitaux. Mais, jusqu'à ce qu'une circulaire vienne préciser début 2008 que les RC éligibles ni à la CMU ni à l'AME pouvaient relever de ce dispositif, trop d'hôpitaux n'imputaient pas toujours les soins sur ce Fonds et préféraient adresser des factures à des familles rom manifestement insolvables, quitte à accumuler des créances jamais réglées.

Tous ces obstacles et dénis de droits participent du mauvais état de santé de la population rom migrante vivant en France. Certes, d'autres causes se conjuguent : problèmes plus anciens de non-accès aux soins dans le pays d'origine; absence d'information et d'éducation à la santé chez des personnes ayant eu un cursus scolaire réduit ; méconnaissance des structures de soins et des démarches; barrière de la langue ; peur des arrestations ; mauvaises conditions de vie liées à la situation précaire de migrant ; ruptures de soins liées à l'instabilité et aux expulsions multiples ; priorités données à la recherche de ressources et de nourriture avant celle de soins. Reste que le climat de suspicion et les multiples pratiques d'entraves des pouvoirs publics à l'accès à une couverture maladie et à l'accès aux soins ont des conséquences négatives en matière de santé publique pour une population qui, pour de nombreuses raisons liées aux conditions de vie, souffre déjà davantage de certaines pathologies (tuberculose, maladies infectieuses, problèmes psychologiques, pathologies materno-infantiles liées à des grossesses précoces ou non suivies, etc.). La situation est si alarmante que, dans son dernier rapport sur la France, le commissaire aux droits de l'homme du Conseil de l'Europe a consacré une partie sur les Roms et a invité les autorités françaises "à garantir un meilleur accès des populations rom aux soins et aux aides médicales ${ }^{(20)}$." Le climat de suspicion qui règne désormais vis-à-vis des citoyens européens sollicitant des droits sociaux n'engage pas dans cette voie. 


\section{Notes}

1. Cet article reprend de nombreux éléments rassemblés dans deux publications récentes : Le droit à la protection sociale des ressortissants communautaires, note pratique, Groupe d'information et de soutien des immigrés (GISTI), octobre 2008, 44 p. (www.gisti.org) ; ROMEUROPE, Rapport 2007-2008, Collectif national droits de l'homme Romeurope, septembre 2008, 172 pages (www.romeurope.org). Larticle se fonde plus particulièrement sur une analyse et un suivi des textes et des pratiques, ainsi que d'un usage pratique du droit (conseils, recours juridiques), de rédaction de guides et de formation en droit à la protection sociale des étrangers.

2. Sur ces questions, voir Mady Denantes, Marie Chevillard, Jean-François Renard, Patrick Flores, "Accès aux soins et inégalités sociales de santé en médecine générale", in La revue française de médecine générale, Volume 20, n85, 2009, p. 22 26.

3. Sur cette histoire dont nous reprenons certains éléments, en ce qui concerne les seuls ressortissants communautaires, voir Antoine Math, "La protection sociale des ressortissants d'États tiers dans l'Union européenne. Vers une citoyenneté sociale de résidence ?", Document de travail IRES, n03.01, juin 2003 (www.ires-fr.org); Antoine Math et Adeline Toullier, "Protection sociale des étrangers. Le difficile chemin vers l'égalité des droits", in Confluences Méditerranée, $\mathrm{n}^{\circ} 48$, Hiver 2003-2004, L'Harmattan, p. 105-119, [résumé "La difficile marche vers l'égalité des droits sociaux", in Hommes et liberté $\mathrm{n}^{\circ} 126$, avril-mai-juin 2004].

4. Circulaire ministérielle ${ }^{\circ} \mathrm{DSS} / \mathrm{DACI} / 2007 / 418$ du 23 novembre 2007 relative au bénéfice de la Couverture maladie universelle de base (CMU) et de la Couverture maladie universelle complémentaire (CMU-C) des ressortissants de l'Union européenne, de l'Espace économique européen et de la Suisse résidant ou souhaitant résider en France en tant qu'inactifs, étudiants ou demandeurs d'emploi.

5. Circulaire DSS/2A/DAS/DPM 2000-239 du 3 mai 2000 relative à la condition de résidence en France prévue pour le bénéfice de la couverture maladie universelle.

6. Point CMU n ${ }^{\circ} 66$, lettre Réseau CNAM du 27 février 2006.

7. Aucune des nombreuses actions ou informations de l'Observatoire du droit à la santé des étrangers (ODSE), qui regroupe une quinzaine d'associations défendant les droits des étrangers en matière d'accès aux soins, ne porte sur des problèmes rencontrés par les ressortissants communautaires (www.odse.eu.org).

8. COMEDE, Guide pratique destiné aux professionnels, Comité médical pour les exilés, 2008, 568 p. (www.comede.org).

9. Par exemple, Adeline Toullier, "Aide médicale d'État : les droits sociaux fondamentaux bafoués", in Droit social, novembre 2005 ; Christel Cournil, "Quand les politiques migratoires françaises 'contaminent' l'accueil sanitaire et l'accès aux soins des étrangers", in Revue trimestrielle des droits de l'homme, $\mathrm{n}^{\circ}$ 72, octobre 2007, p. 1017-1049 ; Didier Maille, Adeline Toullier, Pierre Volovitch, "Comment un droit se vide de son sens faute d'être réellement universel", in Revue de droit sanitaire et social, juillet-août 2005, p. 542-554.

GISTI, "L'accès aux soins des étrangers : débats et évolutions du droit", in Hommes et Migrations, n 1225 , Santé, le traitement de la différence, mai-juin 2000.

10. Rapport sur l'application de la directive 2004/38/CE relative au droit des citoyens de l'Union et des membres de leurs familles de circuler et de séjourner librement sur le territoire des États membres, Parlement européen, janvier 2009

(www.europarl.europa.eu).

11. Le droit à la protection sociale des ressortissants communautaires, Note pratique, Groupe d'information et de soutien des immigrés (GISTI), octobre 2008, 44 p. (www.gisti.org).

12. ROMEUROPE, Rapport 2007-2008, Collectif national droits de l'homme Romeurope, septembre 2008, $172 \mathrm{p}$. (www.romeurope.org).

13. Par exemple, la circulaire ministérielle ${ }^{\circ}$ DSS/DACI/2007/13 du 8 janvier 2007 "relative aux conséquences en matière de sécurité sociale de l'élargissement de l'Union européenne à deux nouveaux États membres (Bulgarie et Roumanie)"

14. Circulaire ministérielle nDSS/DACI/2007/418 du 23 novembre 2007, op. cit.

15. Les autres restrictions supplémentaires introduites en France en 2006 et 2007 pour certaines prestations d'assistance (RMI, API, AAH, CMU de base) concernent des situations relativement marginales.

16. La seule exception, prévue par l'article L. 380-3 du code de la Sécurité sociale, mais rarement rencontrée en pratique, concerne le demandeur d'emploi communautaire entré en France pour chercher du travail et inscrit comme demandeur d'emploi au Pôle emploi (ex-ANPE).

17. Jean-Philippe Lhernould, "Les Européens et la CMU après la circulaire du 23 novembre 2007", in Droit social, février 2008.

18. "Le principe déclaratif en matière de domicile (adresse) s'applique à tous les droits sociaux, y compris l'AME", GISTI, septembre 2008 (www.gisti.org).

19. "Plainte contre la France pour violations du droit communautaire en matière de libre circulation des personnes", Collectif national droits de l'homme, Romeurope, Cimade, GISTI et autres associations, 31 juillet 2008 (www.gisti.org). 20. Mémorandum de Thomas Hammarberg, commissaire aux droits de l'homme du Conseil de l'Europe, faisant suite à sa visite en France du 21 au 23 mai 2008, 20 novembre 2008, Strasbourg, 67 p., (www.coe.int/t/commissioner). 